\title{
Caracterización de aceros dual-phase obtenidos por laminación en caliente $^{(\cdot)}$
}

\author{
A. Monsalve G. , A. Artigas A. , F. Castro C. ${ }^{*}$, R. Colás ${ }^{* *}$ e Y. Houbaert ${ }^{* * *}$
}

Resumen

\begin{abstract}
Se tomaron muestras de acero al C-Mn-Si disponible en el mercado y mediante un proceso de laminación en caliente y bobinado, se obtuvo acero Dual Phase con microestructura y propiedades mecánicas dentro del rango teórico esperado de este material. El proceso termomecánico consistió en producir una fuerte reducción a temperaturas mayores a $\mathrm{A}_{\mathrm{r} 3}$, mediante pasadas de laminación sucesivas, para posteriormente enfriar el acero durante aproximadamente $5 \mathrm{~s}$, (a una velocidad de $20^{\circ} \mathrm{C} / \mathrm{s}$ ) en el rango de equilibrio $\alpha+\gamma$. La temperatura $\mathrm{A}_{\mathrm{r} 3}$, medida mediante calorimetría diferencial de barrido, fue de $890^{\circ} \mathrm{C}$. A continuación se realizó un temple en el rango de temperaturas de bobinado $\left(550-675^{\circ} \mathrm{C}\right)$, enfriando posteriormente las muestras de acuerdo a una curva pre establecida, que corresponde a la curva de enfriamiento real de una bobina. La caracterización microestructural de las muestras obtenidas, se realizó mediante microscopía óptica, microscopía electrónica de barrido y microscopía de fuerza atómica. Adicionalmente se realizó una medición de texturas mediante difracción de rayos X, para el estudio de las orientaciones resultantes, producto de la variación de la temperatura de término de laminación (TTL) y la temperatura de bobinado (TB), determinándose cómo afectan estas variables a las distintas componentes de texturas. Se complementó la información microestructural con los valores de los índices de anisotropía normal y planar, medidos de acuerdo a la norma ASTM E-517. Se correlacionaron las intensidades de las componentes de texturas encontradas con los valores de los índices de anisotropía, encontrándose que sólo es posible producir leves mejoras en el índice de anisotropía normal, a través de una combinación apropiada de las temperaturas de término de laminación y de bobinado.
\end{abstract}

\section{Characterization of dual-phase steels obtained by hot-rolling}

Abstract

Keywords

\section{INTRODUCCIÓN}

Los aceros estructurales han experimentado una creciente mejora en sus propiedades como respuesta a las altas exigencias que el mercado requiere. Equilibrar

\begin{abstract}
Samples were obtained from C-Mn-Si steel available in the market. Through a hot rolling and coiling process, it was possible to obtain Dual-Phase steel with microstructural and mechanical properties in the theoretical range typical of this material. The thermomechanical process consisted of a strong reduction by multiples pass of hot rolling at temperatures above $\mathrm{A}_{\mathrm{r} 3}$, controlled-cooling the sheets during $5 \mathrm{~s}$ (at a rate of $20^{\circ} \mathrm{C} / \mathrm{s}$ in the equilibrium range $\alpha+\gamma$. Temperature $\mathrm{A}_{\mathrm{r} 3}$ measured by differential scanning calorimetry was $890^{\circ} \mathrm{C}$. Quenching was then carried out in the coiling temperatures range $\left(500-675^{\circ} \mathrm{C}\right)$, cooling the samples in accordance to an established curve that corresponds to the actual cooling curve of a coil. The microstructural characterization of the samples obtained was carried out by optical microscopy, scanning electron microscopy and atomic force microscopy. Additionally, texture measurements were carried out by X-ray diffraction in order to study the resulting orientations due to the finishing rolling temperature and coiling temperature, determining the influence on these parameters of the different texture components. The microstructural results were complemented with the normal and planar anisotropy indexes measured in according to the ASTM E-517 standard. The intensities of the different texture components were correlated with the values of anisotropy indexes, finding that it is possible to obtain only a slightly enhancement in the normal anisotropy index through an appropriate combination of finish rolling and coiling temperatures.
\end{abstract}

Steels; Dual-Phase; Hot-rolling; Textures; Drawing.

(•) Trabajo recibido el día 5 de Marzo de 2010 y aceptado en su forma final el día 25 de Junio de 2010.

* Universidad de Santiago de Chile, Depto. Ingeniería Metalúrgica, Av L. Bdo. O’Higgins 3363, Estación Central. Santiago de Chile.

** Facultad de Ingeniería Mecánica y Eléctrica, Universidad Autónoma de Nuevo León, 66451 San Nicolás de los Garza, N.L. México.

*** Department of Metallurgy and Materials Science, University of Ghent, Bélgica. 
demandados en los últimos años, debido al creciente interés en reducir el peso de las estructuras sin comprometer su resistencia mecánica, categoría en que se encuentran los aceros Dual Phase (DP).

Los aceros DP, son aquellos cuya microestructura está compuesta principalmente de ferrita y martensita, encontrándose pequeñas zonas de bainita y austenita estabilizada como resultado del tratamiento térmico. Estos aceros pueden ser obtenidos mediante laminación en caliente, o laminación en frío y recocido contínuo, siendo el tratamiento térmico realizado durante el proceso de fabricación, el que determina las cantidades relativas de las fases presentes. Como características principales de este material, se tiene un comportamiento homogéneo durante la fluencia, una gran zona de endurecimiento por deformación y una elevada ductilidad, conferida por la distribución de las fases presentes.

Recientemente, en estos aceros se han realizado investigaciones orientadas al desarrollo de las texturas durante los procesos de conformado termomecánico ${ }^{[1-3]}$, donde principalmente se ha estudiado la influencia de los parámetros de proceso, como porcentajes de reducción, tiempo entre pasadas de laminación, temperatura de tratamiento, entre otras, en las orientaciones resultantes. Jonas ${ }^{[4} \mathrm{y}^{5]}$, ha sugerido que existe una fuerte correlación entre las características de la austenita (energía de falla de apilamiento, componentes de texturas presentes) y las características de la ferrita obtenida. Esto se explica entre otras cosas, tomando en cuenta las relaciones de epitaxia, que vinculan las componentes de texturas en austenita con las de ferrita. Sin embargo, sigue existiendo un gran campo de investigación en el área de laminación en caliente y la obtención de texturas deseadas en procesos de conformado posteriores.

El objetivo del presente trabajo, es estudiar la influencia de las condiciones de laminación en caliente en las componentes de texturas finales en aceros Dual-Phase, fabricados por laminación en caliente y bobinado.

\section{PROCEDIMIENTO EXPERIMENTAL}

Se tomaron piezas de acero de $145 \mathrm{~mm}$ de largo 65 y $26 \mathrm{~mm}$ de ancho y espesor, respectivamente. La composición química del material inicial se muestra en la tabla I.

Las muestras fueron sometidas a un proceso de laminación en caliente, que consistió en calentar durante una hora a $1.150^{\circ} \mathrm{C}$ y posteriormente laminar la muestra en sucesivas pasadas, calentando la pieza entre pasadas para producir recristalización estática, a una temperatura mayor que $A_{r 3}$. Las temperaturas $A_{r 1}$ y $A_{r 3}$,
Tabla I. Análisis químico

Table I. Chemical analisys

\begin{tabular}{ccccccc}
\hline \multicolumn{6}{c}{ Composición \% Peso } \\
\hline C & Si & Mn & P & S & Al & N \\
\hline 0,067 & 0,83 & 1,53 & 0,017 & 0,0072 & 0,016 & 0,018
\end{tabular}

fueron determinadas mediante calorimetría diferencial de barrido (DSC), obteniéndose 696 y $894^{\circ} \mathrm{C}$ respectivamente. A continuación, el material se enfría en el campo bifásico por aproximadamente $5 \mathrm{~s}$, a una velocidad de aproximadamente $20^{\circ} \mathrm{C} / \mathrm{s}$. Tras este enfriamiento, cada pieza es templada en un baño de sales a una velocidad de aproximadamente $100^{\circ} \mathrm{C} / \mathrm{s}$. El baño de sales se mantuvo a la temperatura de bobinado, es decir, en el rango de 550 a $650^{\circ} \mathrm{C}$, sumergiéndose durante $5 \mathrm{~min}$, con el fin de homogeneizar la temperatura. Este paso se realiza con el objeto de evitar la precipitación de perlita. Esto además es similar a la práctica industrial estándar, consistente en inducir un rápido enfriamiento desde la temperatura de término de laminación, con el objeto de evitar la oxidación de la bobina y la adhesión de las espiras. Posteriormente, la muestra se traslada a un horno de resistencia programado a la misma temperatura del baño de sales, con el objetivo de simular el enfriamiento de la muestra de acuerdo con la curva de enfriamiento real de la bobina. Para eso se emplearon resultados previos de los autores relativos a curvas de enfriamiento de bobinas ${ }^{[6]}$. El esquema del procedimiento se muestra en la figura 1.

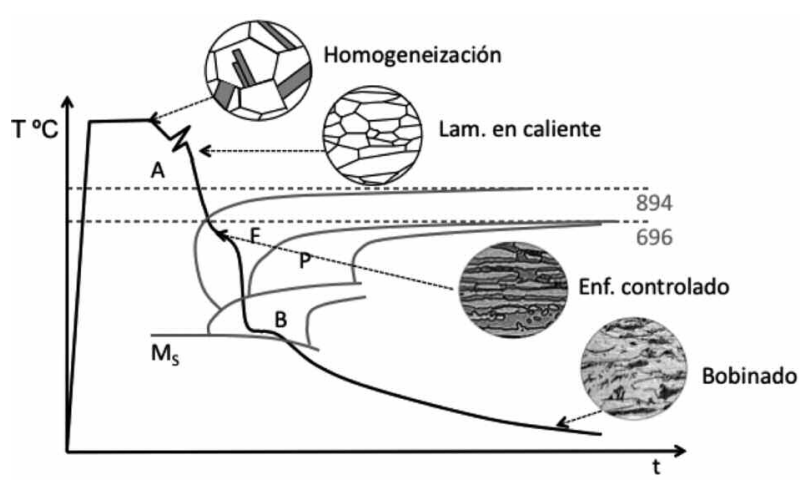

Figura 1. Esquema del tratamiento termo mecánico realizado.

Figure 1. Thermomechanical procedure schedule. 
En la tabla II se detallan los parámetros de laminación utilizados en cada uno de los casos.

Las muestras fueron atacadas con Nital al $2 \%$ para delinear los contornos de grano de la ferrita y con el reactivo Le Pera ${ }^{[7]}$, que permite diferenciar la martensita y la bainita. Se realizó adicionalmente una cuantificación de fases mediante el software Image Pro Plus. La medición de texturas fue realizada en un equipo marca SIEMENS modelo Diffraktometer D5000 que utiliza radiación de molibdeno. Además, para la medición de texturas se utilizó un goniómetro estándar. La microscopía electrónica de barrido se realizó en un microscopio Phillips XL 30 ESEM, equipado con un filamento de $\mathrm{LaB}_{6}$ y operado a $25 \mathrm{kV}$. La microscopía de fuerza atómica, fue realizada en un equipo Veeco modelo Nanoscope $3 \mathrm{~A}$, operado en modo de contacto.

Las muestras observadas por SEM, fueron pulidas y atacadas electrolíticamente. Las muestras observadas por AFM fueron atacadas con nital $2 \%$.

\section{ANÁLISIS Y DISCUSIÓN DE RESULTADOS}

\subsection{Caracterización Mecánica}

En la tabla III se resumen las propiedades de tracción. Para esta prueba se ensayaron 2 probetas por cada muestra laminada, que fueron cortadas en la dirección de conformado y en la dirección perpendicular a la de conformado respectivamente. Con el fin de destacar el comportamiento típico de un acero

Tabla Il. Parámetros de laminación

Table II. Hot rolling parameters

\begin{tabular}{ccccc}
\hline & Muestra & $\mathrm{TA}^{*}{ }^{\circ} \mathrm{C}$ & $\mathrm{TTL}^{* *}{ }^{\circ} \mathrm{C}$ & $\mathrm{TB}^{* * *}{ }^{\circ} \mathrm{C}$ \\
\hline Grupo I & $990 / 550$ & 1.150 & 990 & 550 \\
& $990 / 575$ & 1.150 & 990 & 550 \\
& $900 / 550$ & 1.150 & 900 & 550 \\
& $900 / 575$ & 1.150 & 900 & 575 \\
Grupo II & $990 / 600$ & 1.150 & 990 & 600 \\
& $850 / 600$ & 1.150 & 850 & 600 \\
Grupo III & $840 / 625$ & 1.150 & 840 & 625 \\
& $970 / 650$ & 1.150 & 970 & 650 \\
& $830 / 650$ & 1.150 & 830 & 650 \\
\hline
\end{tabular}

* Temperatura de Austenitización/Soaking temperature.

** Temperatura de Término de Laminación/Finishing rolling temperature.

${ }^{* * *}$ Temperatura de Bobinado/Coiling temperature.
Tabla III. Resumen del promedio de resultados obtenidos del ensayo de tracción

Table III. Tensile test results (average)

\begin{tabular}{ccccccc}
\hline & Probeta & $\begin{array}{c}\sigma_{0} \\
\text { MPa }\end{array}$ & $\begin{array}{c}\text { UTS } \\
\text { MPa }\end{array}$ & $\begin{array}{c}\sigma_{0} l \\
\text { UTS }\end{array}$ & $\begin{array}{c}\varepsilon \\
\%\end{array}$ & $\begin{array}{c}\mathbf{n} \\
(\mathbf{4} \%)\end{array}$ \\
\hline \multirow{4}{*}{ Grupo I } & $990 / 550$ & 314 & 510 & 0,62 & 47 & 0,16 \\
& $990 / 575$ & 373 & 530 & 0,70 & 25 & 0,18 \\
& $900 / 550$ & 383 & 530 & 0,72 & 20 & 0,16 \\
& $900 / 575$ & 304 & 491 & 0,62 & 18 & 0,17 \\
\multirow{2}{*}{ Grupo II } & $990 / 600$ & 314 & 510 & 0,62 & 17 & 0,50 \\
\multirow{2}{*}{ Grupo III } & $850 / 600$ & 383 & 520 & 0,74 & 17 & 0,17 \\
& $840 / 625$ & 363 & 500 & 0,73 & 27,5 & 0,18 \\
& $970 / 650$ & 334 & 481 & 0,69 & 26 & 0,18 \\
& $830 / 650$ & 353 & 510 & 0,69 & 22 & 0,17 \\
\hline
\end{tabular}

DP, se incluye en esta tabla el cuociente $\sigma_{0} /$ UTS. Los datos fueron ordenados en tres grupos de acuerdo a la temperatura de bobinado: el primer grupo corresponde a bajas temperaturas de bobinado, el segundo a temperaturas de bobinado intermedias y el tercero que incluye las altas temperaturas de bobinado. Debido a la influencia que ejerce la temperatura de término de laminación sobre el tamaño de grano, la muestra 990/600 se incluyó dentro del primer grupo, en tanto que la 850/600, fue considerada en el segundo grupo. Esta diferencia se aclara más adelante al analizar los tamaños de grano obtenidos.

En la figura 2 se muestran algunas curvas de tracción, pudiéndose apreciar el comportamiento clásico de los aceros DP, es decir, una gran diferencia entre

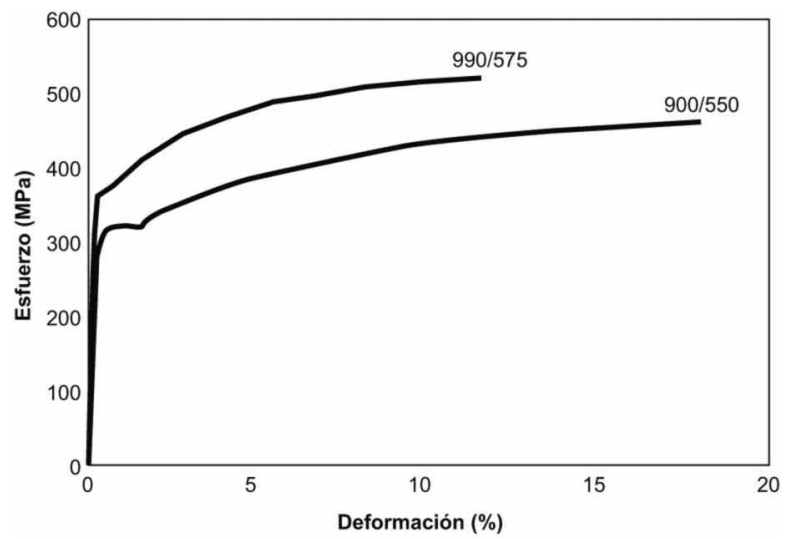

Figura 2. Curvas esfuerzo-deformación ingenieril para algunas muestras.

Figure 2. Some engineering stress-strain curves. 
el esfuerzo de fluencia y el esfuerzo tensil máximo y la ausencia de fluencia heterogénea. En general estos resultados se encuentran en el rango de los aceros HSLA $^{[8]}$

Se observó que el comportamiento homogéneo en fluencia se dio solamente a las temperaturas de bobinado en el rango de 550 a $600^{\circ} \mathrm{C}$. Las muestras bobinadas desde 625 a $650^{\circ} \mathrm{C}$ mostraron fluencia heterogénea. La razón de esto, es que no se encontró martensita en aquellas muestras bobinadas en el rango 625 a $650^{\circ} \mathrm{C}$, formándose microconstituyentes como bainita y perlita en lugar de martensita. La figura 3 muestra una comparación entre la curva obtenida de la muestra 990/575 (dentro del rango DP) y la curva 840/625 (fuera del rango DP), donde se aprecia la diferencia existente entre las zonas de fluencia.

Los resultados obtenidos para los índices de anisotropía normal $(\bar{r})$ y planar $(\Delta \bar{r})$ se muestran en la tabla IV. Aunque son relativamente bajos, la mayor parte de ellos están en el rango de los aceros al carbono laminados.

\subsection{Caracterización Metalográfica}

Como se muestra en la figura 4, todas las probetas analizadas en los rangos de temperatura de bobinado desde 550 a $650{ }^{\circ} \mathrm{C}$ muestran regiones claras y oscuras (ataque con reactivo Le Pera). Las zonas color marrón corresponden a ferrita y como se aprecia en

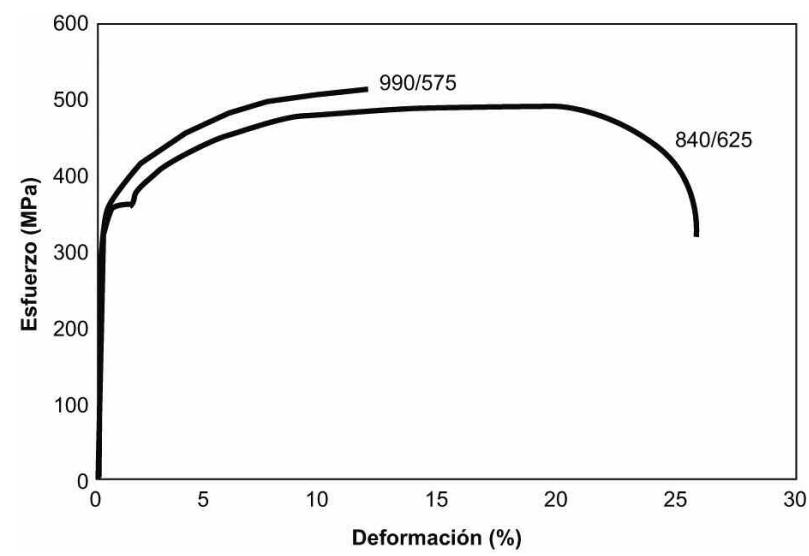

Figura 3. Curvas de tracción de las muestras $990 / 575$ y $840 / 625$. Se observa el comportamiento clásico de los aceros DP en comparación al comportamiento de los aceros al carbono normales.

Figure 3. Tensile curves of samples 990/575 and 840/625 showing classical DP behaviour compared with plain carbon steel.
Tabla IV. Resultados de ensayos de anisotropía

Tabla IV. Anisotropy test results

\begin{tabular}{lccr}
\hline & & $\bar{r}$ & \multicolumn{1}{c}{$\Delta \boldsymbol{r}$} \\
\hline \multirow{3}{*}{ Grupo I } & $990 / 550$ & 0,61 & $-0,52$ \\
& $990 / 575$ & 0,91 & 0,05 \\
& $900 / 550$ & 0,84 & 0,33 \\
\multirow{2}{*}{ Grupo II } & $900 / 575$ & 0,49 & $-0,19$ \\
Grupo III & $990 / 600$ & 0,44 & $-0,27$ \\
& $850 / 600$ & 0,72 & 0,21 \\
& $840 / 625$ & 0,87 & 0,34 \\
& $930 / 650$ & 0,96 & $-0,07$ \\
& $830 / 650$ & 0,95 & 0,43 \\
\hline
\end{tabular}

las imágenes, es esta fase la que se encuentra en mayor proporción. Las zonas sin atacar (claras), corresponden a martensita y las zonas color marrón oscuro corresponden a bainita.

En la tabla V se muestra un resumen con las observaciones metalográficas realizadas (ataque con nital

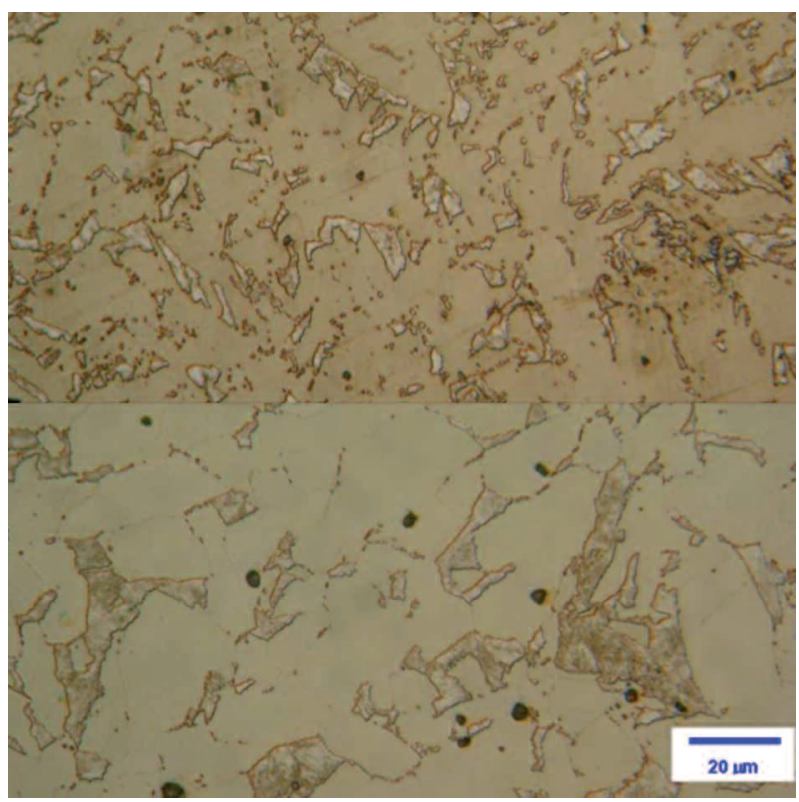

Figura 4. Micrografías de diferentes muestras obtenidas; 900/550 (superior) y 830/650 (inferior). Se observan zonas de ferrita (marrón claro), bainita o perlita (marrón oscuro) y martensita (blanco). Atacado con reactivo LePera.

Figure 4. Micrographs of samples 900/550 (upper) y 830/650 (lower) showing ferrite (light brown), bainite or pearlite (dark brown) and martensite (white). LePera etching. 
Tabla V. Resumen de datos microestructurales

Table V. Microstructural data

\begin{tabular}{|c|c|c|c|c|c|c|}
\hline & \multirow[b]{2}{*}{ Muestras } & \multirow{2}{*}{$\begin{array}{l}\text { Tamaño } \\
\text { de Grano } \\
\text { ASTM }\end{array}$} & \multirow{2}{*}{$\begin{array}{c}\text { Tamaño } \\
\mu m\end{array}$} & \multicolumn{3}{|c|}{ Fases Presentes, \% } \\
\hline & & & & Ferrita & $\begin{array}{c}\text { Marten- } \\
\text { sita }\end{array}$ & $\begin{array}{c}\text { Bainita+ } \\
\text { Perlita }\end{array}$ \\
\hline \multirow{5}{*}{ Grupo I } & $0 / 550$ & $9-10$ & $15,9-11,2$ & 81 & 3 & 16 \\
\hline & $990 / 575$ & $9-10$ & $15,9-11,2$ & 86 & . & t \\
\hline & $990 / 600$ & $9-10$ & $15,9-11,2$ & 86 & 2 & 12 \\
\hline & $900 / 550$ & $9-10$ & $15,9-11,2$ & 81 & 5 & 14 \\
\hline & $900 / 575$ & $9-10$ & $15,9-11,2$ & 80 & 4 & 16 \\
\hline \multirow{2}{*}{ Grupo II } & $850 / 600$ & $8-9$ & $22,5-15,9$ & 84 & 1 & 15 \\
\hline & $840 / 625$ & $8-9$ & $22,5-15,9$ & 86 & 0 & 14 \\
\hline \multirow[t]{2}{*}{ Grupo III } & $\begin{array}{l}970 / 650 \\
830 / 650\end{array}$ & $\begin{array}{l}6-7 \\
78\end{array}$ & $\begin{array}{l}44,9-31,8 \\
31,225\end{array}$ & 88 & 0 & 12 \\
\hline & $830 / 650$ & 7-8 & $31,8-22,5$ & 86 & 0 & 14 \\
\hline
\end{tabular}

$2 \%$ ), apreciándose el tamaño de grano ferrítico y los porcentajes de las distintas fases presentes. El nivel de las inclusiones de cada muestra fue catalogado según la norma ASTM E - 45 y para todas las muestras fue de $1 \mathrm{~B}$.

La microestructura resultante consiste principalmente de ferrita y pequeñas zonas dispersas en la matriz de martensita, bainita y perlita. En algunas muestras se detectó mediante SEM en la modalidad de difracción de electrones, la presencia de austenita, pero no fue posible resolverla mediante microscopía óptica. La ferrita es principalmente equiaxial, sin embargo, en las muestras templadas a más bajas temperaturas $\left(550\right.$ y $\left.575^{\circ} \mathrm{C}\right)$ se encontró ferrita del tipo Widmanstätten $\left(\alpha_{\mathrm{W}}\right)$, como se muestra en la figura 5 .

Las otras fases presentes en la microestructura (martensita, cementita, etc.) se distribuyeron de manera relativamente homogénea a lo largo del material, encontrándose algunas zonas bandeadas donde la concentración de segundas fases fue mayor (Fig. 6 a)). Esto se atribuye a la segregación de manganeso en el material que causa un aumento local en la templabilidad difundiendo el carbono hacia esos sitios al haber tiempo suficiente (mayores temperaturas de temple $)^{[9-11]}$. En las muestras templadas a baja temperatura $\left(550\right.$ a $\left.600{ }^{\circ} \mathrm{C}\right)$ no se observa una estructura bandeada (Fig. 6b)). Esto debido a que a bajas temperaturas de temple, el tiempo es demasiado corto como para permitir la difusión de carbono de largo alcance, que normalmente es lo que evidencia el bandeado. Además, como se mencionó en el párrafo anterior, a altas temperaturas de temple, la ferrita aparece equiaxial, en tanto que a bajas temperaturas de temple, predomina la morfología de placas.
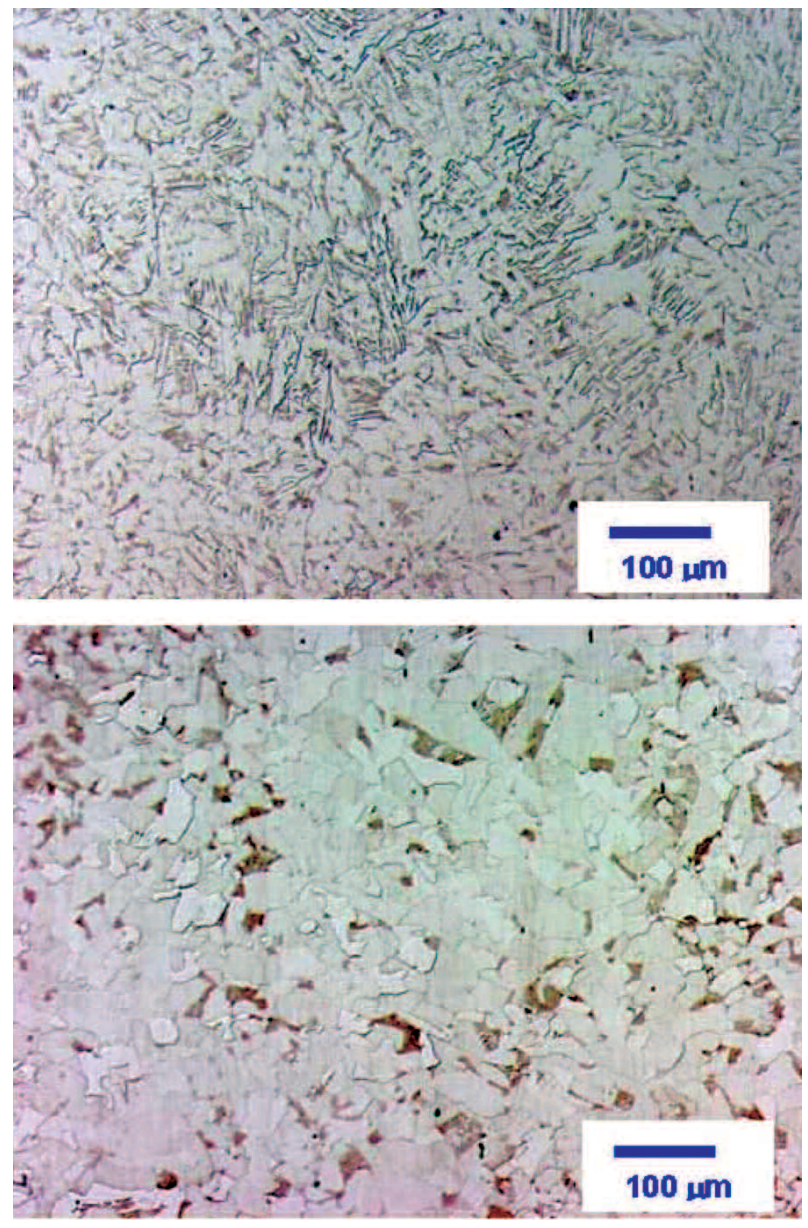

Figura 5. Micrografías de las muestras a) $900 / 550$ y b) $970 / 650$. Atacado con nital.

\section{Figure 5. Micrographs taken from samples a) $900 / 550$ and b) $970 / 650$. Nital etching.}

En las muestras templadas a temperaturas iguales o superiores a $600^{\circ} \mathrm{C}$ se detectó la presencia de perlita gruesa en la microestructura en cantidades no mayores de $2 \%$.

Las muestras templadas a más bajas temperaturas (Grupo I), presentan los menores tamaños de grano, en tanto que las muestras templadas a temperaturas intermedias (Grupo II), presentan mayores tamaños de grano que las del Grupo I. A su vez, los mayores tamaños de grano se presentaron en aquellas muestras templadas a más altas temperaturas (Grupo III). Esto muestra la influencia que ejerce la temperatura de temple sobre el tamaño de grano ferrítico. Esto es consistente con el hecho de que a medida que disminuye la temperatura de bobinado, aumenta el sobreenfriamiento, con lo que se producen mayor cantidad de núcleos de ferrita con el consiguiente menor tamaño de grano. Además, a menores temperaturas de bobinado, aparecen estructuras aciculares que contribuyen a afinar dicho tamaño de grano. 

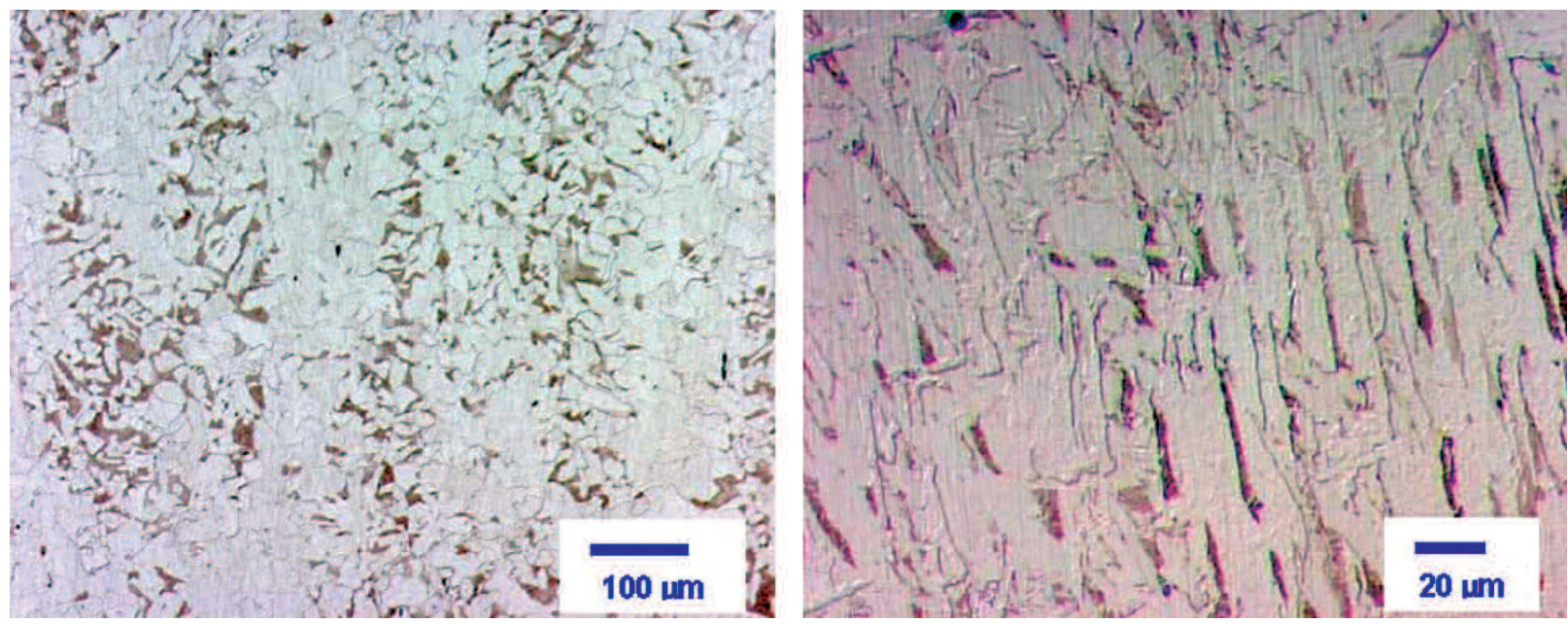

Figura 6. Imagen de la muestra, a) 830/650 y b) 900/550. Atacada con Nital.

Figure 6. Images of samples, a) 830/650 and b) 900/550. Nital etching.

La figura 7 muestra la microestructura revelada con el ataque LePera ${ }^{[7]}$, de martensita (zona clara) y bainita (zona oscura).

Los diferentes aspectos microestructurales pueden además explicar algunos aspectos relativos a las propiedades mecánicas. Probablemente los microconstituyentes distintos de ferrita (martensita, perlita y bainita), tienen un efecto similar en la resistencia máxima del material ${ }^{[8 \text { y } 12]}$, aumentando el valor de esta propiedad mecánica de acuerdo a la fracción presente en el acero. Esto se puede sustentar observando los valores de la resistencia máxima y la cuantificación de fases para las distintas muestras. Al sumar las fracciones de martensita, perlita y bainita, se obtiene en general que los mayores valores de resistencia máxima, están asociados a los mayores valores en la fracción de estos microconstituyentes, tal como se aprecia en la figura 8.

Se observó mediante microscopía electrónica de barrido, que la morfología de la martensita presenta

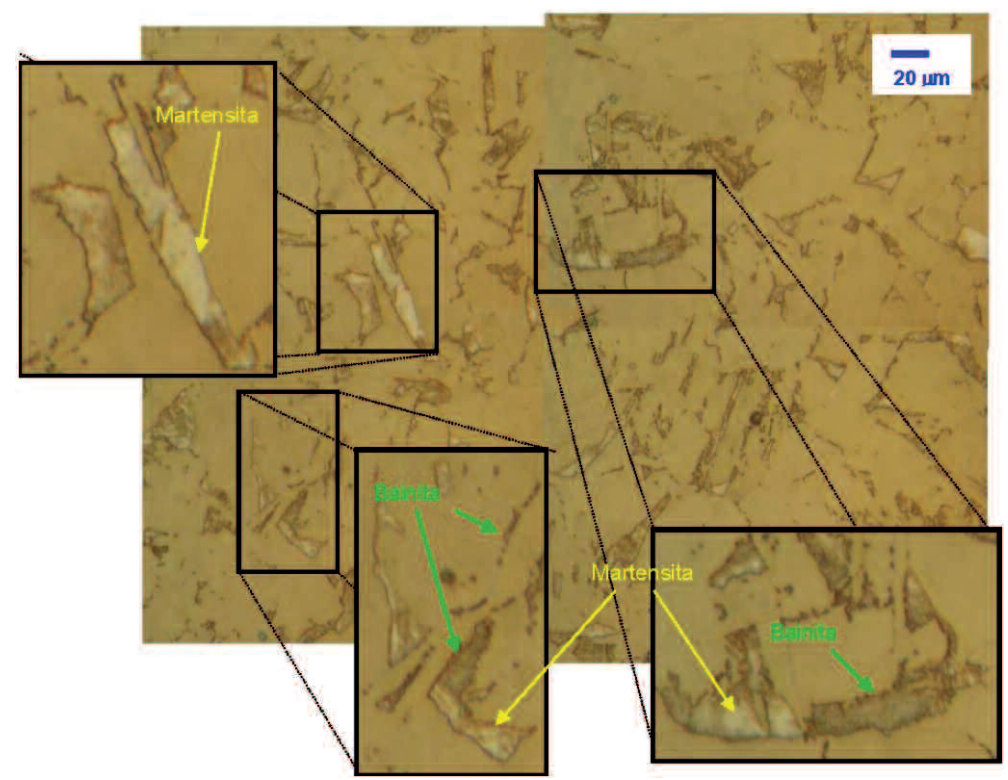

Figura 7. Imagen de la microestructura de la muestra 990/575. Atacado con LePera.

Figure7. Microstructure of sample 990/575. LePera etching. 


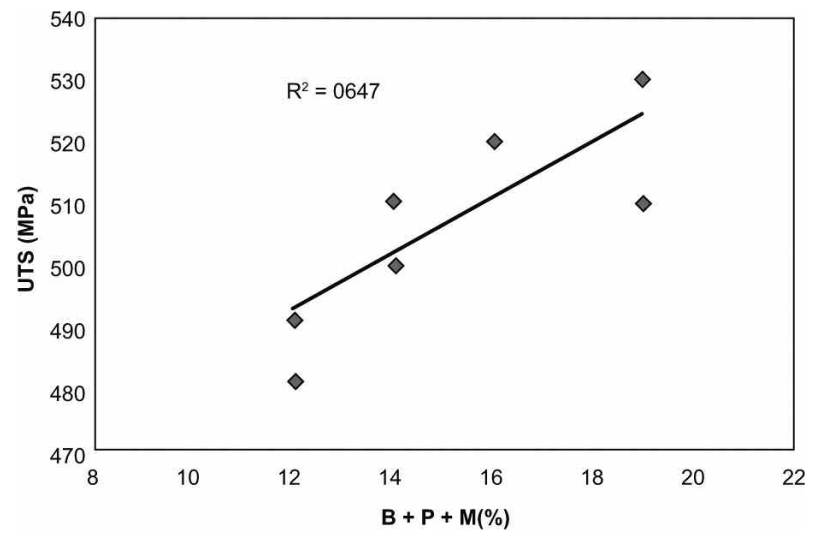

Figura 8. Máxima resistencia a la tracción versus porcentaje de bainita más perlita más martensita.

Figure 8. Maximum tensile strength versus percent of bainite plus pearlite plus martensite.

una forma bastante particular. La figura 9 muestra lo que parecen ser placas alargadas muy similares a la

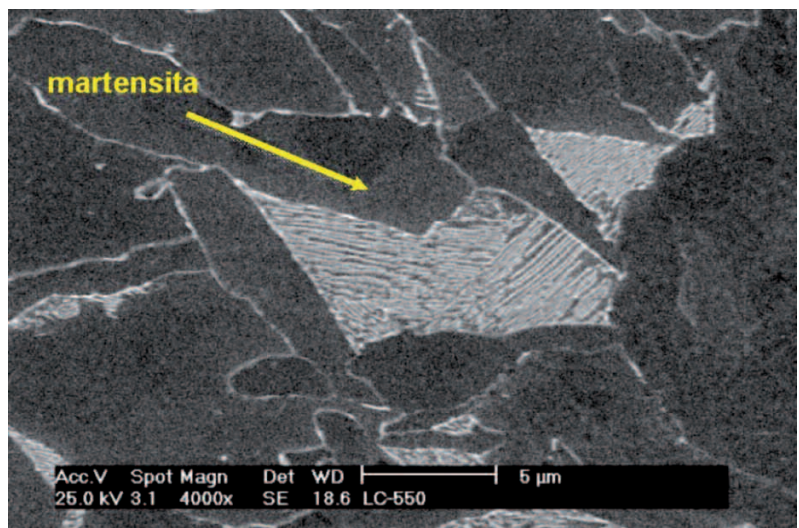

Figura 9. Imagen de muestra 900/550 donde se aprecia Martensita (flecha). Atacado con Nital.

Figure 9. SEM image of sample 900/550. The arrow points martensite. Nital etching.

perlita. Mediante la técnica de microscopía de fuerza atómica, figura 10 , se detectaron zonas similares a las descritas anteriormente.

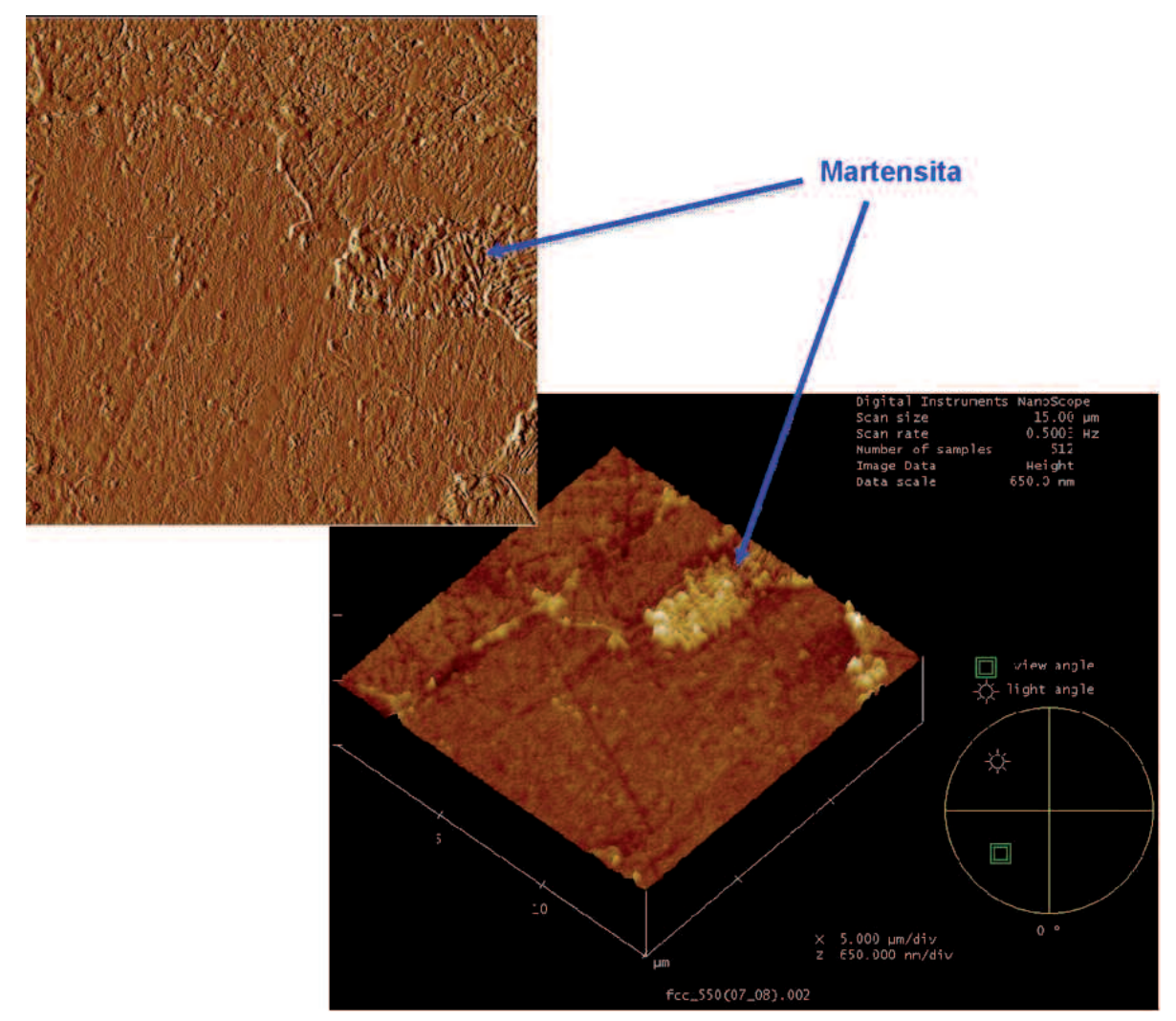

Figura 10. Mapa de fuerza atómica de la muestra 900/550 donde se observa una zona de martensita (flecha).

Figure 10. AFM image of sample 900/550. The arrow points corresponds to martensite. 


\subsection{Estudio de orientaciones}

En el estudio de las diferentes orientaciones obtenidas en ferrita, debe tenerse presente que durante la transformación de austenita a ferrita se deben cumplir las relaciones de Kurdjumov y Sachs. De acuerdo a este modelo, una orientación en austenita se transforma en 24 variantes en ferrita ${ }^{[13]}$. A su vez, la austenita recristalizada presenta fundamentalmente la componente de textura cubo que se transforma en ferrita en la componente $\{100\}\langle 110\rangle_{\alpha}$. Si la transformación de austenita a ferrita tiene lugar a bajas temperaturas, la austenita deformada dará origen a una mezcla de componentes, entre las cuales aparecerán la componente latón, cobre, azufre y Goss ${ }^{[4]}$.

\subsubsection{Influencia de la Temperatura de Bobinado}

En la figura 11 se muestran las FDO para $\varphi_{2}=45^{\circ}$ de las muestras (a) 990/575 y (b) 970/650. Dado que las TTL son aproximadamente las mismas y las TB son muy distintas, pueden considerarse como representativas para el estudio de la variación de las componentes de textura con la temperatura de bobinado. Se aprecia que a temperaturas de bobinado bajas, la intensidad de la componente de textura $\{100\}\langle 110\rangle_{\alpha}$ es levemente mayor que a temperaturas altas, notándose que dicha componente decrece hasta casi desaparecer en la muestra bobinada a $650^{\circ} \mathrm{C}$. Sin embargo, la ocurrencia de esta componente es atribuible a la temperatura de término de laminación que es relativamente alta. Se sabe ${ }^{\left[4 \text { y }^{5]}\right.}$, que esta componente de textura es producto de la transformación de la austenita recristalizada de orientación $\{100\}\langle 001\rangle_{\gamma}$. En cuanto a la intensidad de la fibra $\gamma$, las diferencias observadas entre bajas y altas temperaturas de bobinado, no son concluyentes.

Considerando el análisis anterior y la tabla IV, el comportamiento del índice de anisotropía promedio observado en las muestras es bajo en comparación con los aceros al carbono. La razón por la cual el índice $\bar{r}$ es bajo se encuentra en la débil texturización del material según la fibra $\gamma$ y por la relativa mayor intensidad de la componente $\{001\}\langle 110\rangle_{\alpha}$.

Los valores de $\Delta \mathrm{r}$ obtenidos son en general dispersos, encontrándose valores cercanos a cero para aquellas muestras con valores más altos del índice $r$, específicamente las muestra 990/575 y 970/650.

\subsubsection{Influencia de la Temperatura de Término de Laminación}

En la figura 12 se muestran las FDO para $\varphi_{2}=45^{\circ}$ de las muestras (a) 970/650 y (b) $830 / 650$. Dado que las TB son las mismas y las TTL son muy distintas, pueden considerarse como representativas para el estudio de la variación de las componentes de textura con la temperatura de término de laminación.

En la figura 12 se puede observar que la componente de textura $\{001\}\langle 110\rangle_{\alpha}$ aparece con una intensidad levemente mayor en aquella muestra con TTL de $970^{\circ} \mathrm{C}$, en tanto que en el caso de la muestra con TTL de $860^{\circ} \mathrm{C}$, dicha componente de textura no aparece. Esta componente está relacionada con la componente $\{100\}\langle 001\rangle$, de la austenita recristalizada $^{[14-16]}$, lo que podría indicar que en la muestra

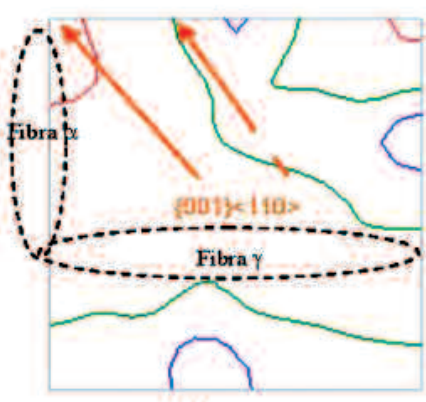

(a)

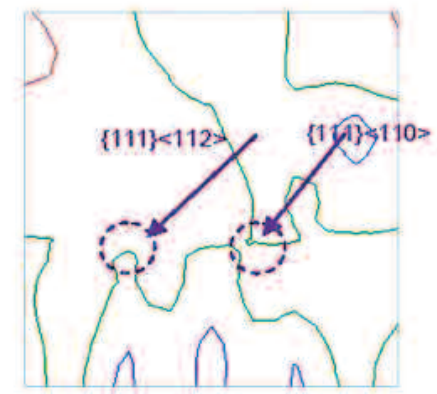

(b)

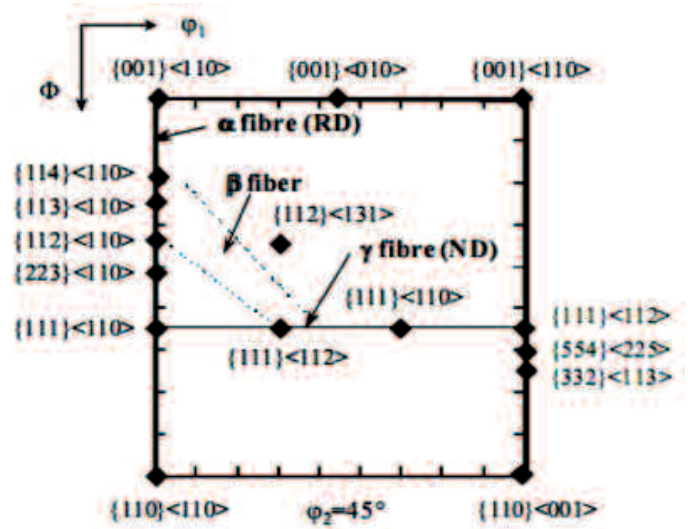

Figura 11. Comparación entre FDO en $\varphi_{2}=45^{\circ}$, a) 990/575 y b) 970/650. Azul: 0,6; Verde: 1,0; Rojo: 1,7 .

Figure 11. Comparison between ODF in $\varphi_{2}=45^{\circ}$, a) 990/575 y b) 970/650. Blue: 0.6; Green: 1.0; Red: 1.7. 


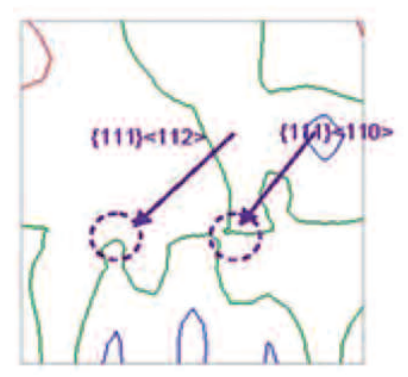

(a)

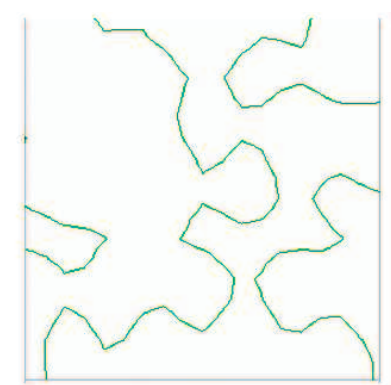

(b)

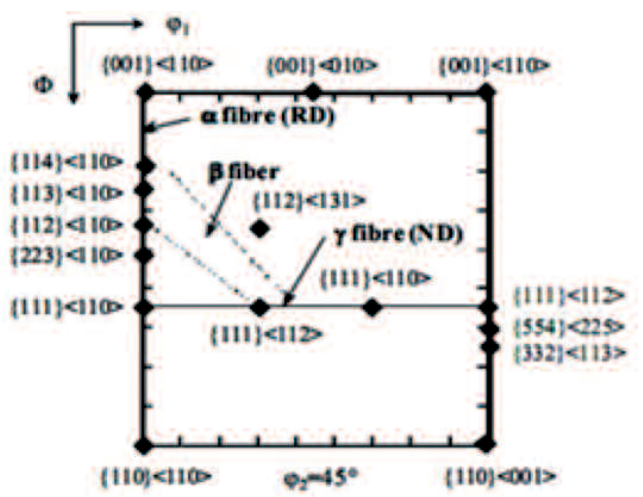

Figura 12. Comparación entre FDO en $\varphi_{2}=45^{\circ}$, a) 970/650, b) 830/650. Azul: 0,6; Verde: 1,0; Rojo: 1,7 .

Figure 12. Comparison between ODF en $\varphi_{2}=45^{\circ}$, a) 970/650, b) 830/650. Blue: 0.6; Green: 1.0; Red: 1.7.

con TTL de $970{ }^{\circ} \mathrm{C}$ podría encontrarse austenita recristalizada, en tanto que en la muestra cuya TTL es de $860^{\circ} \mathrm{C}$, hay una fracción inferior de austenita recristalizada. Para confirmar esta hipótesis, debe realizarse la determinación experimental de la temperatura de no recristalización de la austenita. Este punto constituye una línea de trabajo futuro a desarrollar en la presente investigación.

En ambos casos de TTL, se observan las componentes de textura $\{111\}\langle 110\rangle_{\alpha}$ y $\{111\}\langle 112\rangle_{\alpha}$ casi con una intensidad unitaria, lo que permite concluir que el efecto de distintas TTL en las componentes de texturas resultantes no es significativo.

Debido a que la presencia de texturas benéficas para el proceso de embutición profunda es baja, se puede concluir que el comportamiento del material ante la embutición no será satisfactorio.

Con el fin de facilitar el análisis de la dependencia del índice de anisotropía con las variables de proceso, en la figura 13 se muestra la variación de dicho índice con la temperatura de bobinado, para bajas TTL (inferiores a $900^{\circ} \mathrm{C}$ ), pudiendo apreciarse el incremento en el valor de este índice a medida que aumenta la TB. Esta correlación presenta un factor de correlación de 0,97 , por lo que es estadísticamente confiable. La razón de esto puede estar relacionada con las componentes de texturas favorables a la embutición que se generan al transformarse la austenita deformada en ferrita, específicamente las componentes $\{332\}\langle 113\rangle_{\alpha}$ y $\{113\}\langle 110\rangle_{\alpha}$.Para TTL superiores a $900^{\circ} \mathrm{C}$, no se encontró un ajuste con un grado aceptable de correlación, razón por lo cual, dichos casos no fueron incluidos en esta figura.

Lo anterior permitiría concluir que, con el fin de lograr mayores valores en el índice de anisotropía normal, es aconsejable aumentar la temperatura de

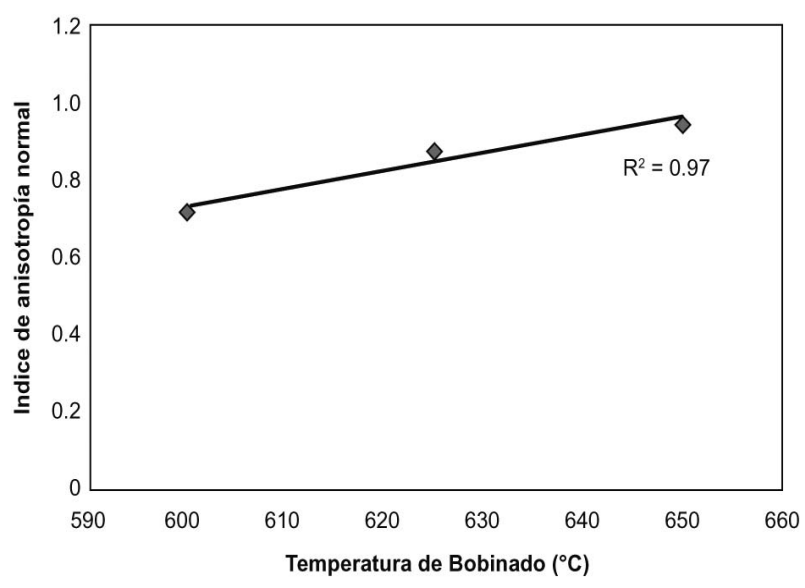

Figura 13. Variación del índice r promedio, con la temperatura de bobinado para muestras con bajas temperaturas de término de laminación.

Figure 13. Graph showing average rv/s coiling temperature for samples with low finish rolling temperature.

bobinado y utilizar temperaturas de término de laminación bajas.

\section{CONCLUSIONES}

- Se estudiaron las características mecánicas y microestructurales de un acero Dual Phase, obtenido mediante un proceso de laminación en caliente a partir de un acero comercialmente disponible.

- El rango de propiedades mecánicas se encuentra en el orden de los aceros HSLA laminados en 
caliente, sin embargo, el índice de anisotropía es considerado bajo.

- Las mediciones de textura tampoco muestran que el material estudiado sea elegible para un proceso de embutición.

- Es posible aumentar levemente el valor del índice de anisotropía normal, aumentando la temperatura de bobinado y bajando la temperatura de término de laminación.

- Se observó que las principales orientaciones presentes en las muestras, fueron las $\{100\}\langle 110\rangle_{\alpha}$, $\{111\}\langle 110\rangle_{\alpha},\{111\}\langle 112\rangle_{\alpha}$.

\section{Agradecimientos}

Los autores desean agradecer a los proyectos FON DECYT № 11080003 y №1060008 por el apoyo financiero para realizar la presente investigación, a la DICYT USACH por el apoyo presentado, a CONACYT (México) y al proyecto bilateral entre los gobiernos de Bélgica y el de Chile.

\section{REFERENCIAS}

[1] B. Gardey, S. Bouvier, V. Richard y B. Bacroix, Mater. Sci. and Eng. A 400-401 (2005) 136141.

[2] A. Kumar, S. B. Singh y K.K. Ray, Mater. Sci. and Eng. A 474 (2008) 270-282.
[3] R. Petrov, L. Kestens, A. Wasilkowska e Y. Houbaert, Mater. Sci. Eng. A 447 (2007) 285-297.

[4] R. K. Ray y J.J. Jonas, Inter. Mater. Rev. 35 (1990) 1-36.

[5] R. K. Ray, J.J. Jonas y R.R. Hook, Inter. Mater. Rev. 39 (1994) 129-172.

[6] A. Monsalve, A. Artigas, D. Celentano y F. Meléndez, Rev.Metal.Madrid (2004) 193-198.

[7] F. S. LePera, J.l of Met. 32 (1980) 38-39.

[8] T. Waterschoot, L. Kestens y B.C. De Cooman, Mat. Met. Trans. A 33 (2002) 1.091-1.102.

[9] C. A. Charles, Mat. Sc. and Tech. 14 (1998) 496-503.

[10] A. Sakir, ISIJ International 31 (1991) 1.445 1.446.

[11] Y. Tomita, Mat. Sc. and Tech 11 (1995) 994997.

[12] V. B. Ginzburg y R. Ballas, "Flat rolling fundamentals", $1^{\underline{a}}$ Ed. Marcel Dekker, New York, EE.UU. 2000.

[13] J. Savoie, R.K. Ray, M. P. Butrón-Guillén y J.J. Jonas, Acta Metall. Mater. 42 (1994) 2.5112.523 .

[14] S. Sun y M. Pugh, Mater. Sci. Eng. A 335 (2002) 298-308.

[15] T. Ros-Yáñez e Y. Houbaert, A. Mertens, Mater. Charac. 47 (2001) 93-104.

[16] A. García-Junceda, F.G. Caballero, C. Capde vila y C. García De Andrés, Scrip. Mater. 57 (2007) 89-92. 